Pacific Journal of Mathematics

CONJUGATES OF EQUIVARIANT HOLOMORPHIC MAPS OF

MIN Ho LeE 


\title{
CONJUGATES OF EQUIVARIANT HOLOMORPHIC MAPS OF SYMMETRIC DOMAINS
}

\author{
MiN Ho LEE
}

\begin{abstract}
In this paper we construct the conjugates of equivariant holomorphic maps of symmetric domains associated to morphisms of arithmetic varieties. We also prove that the conjugate of a Kuga fiber variety is another Kuga fiber variety.
\end{abstract}

0. Introduction. Let $\mathbf{G}$ be a simply connected semisimple algebraic group over $\mathbf{Q}$ that does not contain direct factors defined over $\mathbf{Q}$ and compact over $\mathbf{R}$, and let $K$ be a maximal compact subgroup of the semisimple Lie group $G=\mathbf{G}(\mathbf{R})$. We assume that the symmetric space $D=G / K$ has a complex structure. Let $\Gamma$ be a torsion free arithmetic subgroup of $\mathbf{G}$ and let $X=\Gamma \backslash D$ be the corresponding arithmetic variety. For each $\sigma \in \operatorname{Aut}(X)$ it is known (cf. [5], [6], [7], [10]) that the conjugate $X^{\sigma}$ of $X$ is also an arithmetic variety.

Let $\mathbf{G}^{\prime}$ be another semisimple algebraic Q-group, and consider the corresponding objects $G^{\prime}, K^{\prime}, D^{\prime}, \Gamma^{\prime}$ and $X^{\prime}$ as in the case of $\mathbf{G}$. Let $\rho: G \rightarrow G^{\prime}$ be a homomorphism of Lie groups and $\tau: D \rightarrow D^{\prime}$ a holomorphic map such that $(\rho, \tau)$ is an equivariant pair and $\rho(\Gamma) \subset \Gamma^{\prime}$. Then $\tau$ induces the morphism $\phi: X \rightarrow X^{\prime}$ of arithmetic varieties. Let $D^{\sigma}$ and $D^{\prime \sigma}$ be the universal covering spaces of $X^{\sigma}$ and $X^{\prime \sigma}$ respectively, and let $\tau^{\sigma}: D^{\sigma} \rightarrow D^{\prime \sigma}$ be the lifting of $\phi^{\sigma}: X^{\sigma} \rightarrow X^{\prime \sigma}$. Let $G_{0}$ and $G_{0}^{\prime}$ be the connected components of the identity of $\operatorname{Aut}\left(D^{\sigma}\right)$ and $\operatorname{Aut}\left(D^{\prime \sigma}\right)$ respectively. If $\Gamma^{\sigma} \subset G_{0}$ and $\Gamma^{\prime \sigma} \subset G_{0}^{\prime}$ are the fundamental groups of $X^{\sigma}$ and $X^{\prime \sigma}$ respectively, then we have the following result, Theorem 5.2 of this paper.

THEOREM. There exist a finite covering $G_{1}^{\sigma}$ of $G_{0}^{\sigma}$ and a homomorphism $\rho_{1}^{\sigma}: G_{1}^{\sigma} \rightarrow G_{0}^{\prime \sigma}$ such that $\rho_{1}^{\sigma}$ and $\tau^{\sigma}$ are equivariant and $\rho_{1}^{\sigma}\left(\Gamma^{\sigma}\right)$ is contained in $\Gamma^{\sigma}$.

As an application of this result we consider the conjugates of Kuga fiber varieties. Let $\mathbf{G}^{\prime}=\operatorname{Sp}(V, \beta)$ for some $\mathbf{Q}$-vector space $V$ and a nondegenerate alternating bilinear form $\beta$, and assume that $X=\Gamma \backslash D$ is compact. Then from the equivariant pair $(\rho, \tau)$ we can construct a 
Kuga fiber variety $\pi: Y \rightarrow X$ which is a fiber bundle such that $X$ and $Y$ are complex projective varieties, $\pi$ is a morphism of varieties, and the fibers are polarized abelian varieties (see $\S 6$ for details). For each $\sigma \in \operatorname{Aut}(\mathbf{C})$ we obtain the conjugate $\pi^{\sigma}: Y^{\sigma} \rightarrow X^{\sigma}$ of the Kuga fiber variety $\pi: Y \rightarrow X$. Then we have the following theorem, Theorem 6.3 of the text.

THEOREM. $\pi^{\sigma}: Y^{\sigma} \rightarrow X^{\sigma}$ is a Kuga fiber variety.

The above theorem is known when $\sigma: Y \rightarrow X$ is a family of abelian varieties associated to a PEL-type (cf. [14]) and it is also proved in [9] for Kuga fiber varieties constructed under certain assumptions. The theorem is also an immediate consequence of the main theorems in [5] and [10] in the case that $\phi$ arises from a homomorphism $\tilde{\rho}: \mathbf{G} \rightarrow \mathbf{G}^{\prime}$ of algebraic groups defined over $\mathbf{Q}$ and $\tau$ maps the CM-points of $D$ to the CM-points of $D^{\prime}$. In general, however, $\tau$ does not necessarily map the CM-points to CM-points (see [5, Proposition 1.11] for a necessary and sufficient condition for $\tau$ to map CM-points to CM-points).

This paper contains a part of the results in my Ph.D. thesis. I would like to thank my thesis advisor Professor Michio Kuga. I would also like to thank the referee for various helpful suggestions.

1. Prouniversal covering manifolds. In this section we shall review some of the results in [6] and [7]. Let $\mathbf{G}$ be a simply connected semisimple algebraic group over $\mathbf{Q}$ that does not contain direct factors defined over $\mathbf{Q}$ and compact over $\mathbf{R}$, and let $G=\mathbf{G}(\mathbf{R})$ be the group of real elements of $\mathbf{G}$. If $K$ is a maximal compact subgroup of $G$, we assume that the associated symmetric space $D=G / K$ has a $G$ invariant complex structure.

Let $\Gamma$ be an arithmetic subgroup of $\mathbf{G}$ that does not contain elements of finite order. Then the quotient space $X=\Gamma \backslash D$ has a natural structure of a complex manifold. Such complex manifolds are called arithmetic varieties. By a theorem of Baily and Borel ([3]) $X$ has a structure of an algebraic variety over $\mathbf{C}$, and this structure is unique by a theorem of Borel ([4]).

Let $\left\{\Gamma_{k} \mid k=1,2,3, \ldots\right\}$ be an inductive system of subgroups of finite index of $\Gamma$ such that each $\Gamma_{k}$ is an arithmetic subgroup and

$$
\Gamma_{k} \subset \Gamma_{j} \text { for } j<k \text {. }
$$

Then, for each $k$, the quotient space $X_{k}=\Gamma_{k} \backslash D$ is an arithmetic variety which is a finite unramified covering manifold of $X$, and the 
collection $\left\{X_{k} \mid k \geq 1\right\}$ is a projective system of finite unramified covering manifolds of $X$. The projective limit

$$
\widehat{D}=\lim X_{k}
$$

has a natural structure of a non-connected complex manifold, which does not depend on the representation of $\widehat{D}$ as a projective limit. If

$$
\hat{x}=\lim _{\longleftarrow} x_{k}
$$

is an element of $\widehat{D}$ with $x_{k} \in X_{k}$ for each $k$, then the open neighborhoods of $\hat{x}$ in $\hat{D}$ are determined as follows: If $\left\{U_{k}\right\}$ is a collection of open sets $U_{k} \subset X_{k}$ containing $x_{k}$ for each $k$, then the open neighborhood of $\hat{x}$ associated to $\left\{U_{k}\right\}$ is given by

$$
N\left(\hat{x},\left\{U_{k}\right\}\right)=\left\{\hat{y}=\lim _{k} y_{k} \in \widehat{D} \mid y_{k} \in U_{k} \text { for all } k\right\} .
$$

Let $\pi: D \rightarrow X$ and $\pi_{k}: X_{k} \rightarrow X$ be the canonical projections. To construct a mapping $\hat{\mu}: D \rightarrow \widehat{D}$, we fix a point $x \in X$ and two elements $d_{0} \in D$ and $\hat{d}_{0} \in \widehat{D}$ such that

$$
\pi\left(d_{0}\right)=\hat{\pi}\left(\hat{d}_{0}\right)=x .
$$

Then for each $k$ there is a unique map $\mu_{k}: D \rightarrow X_{k}$ such that $\pi_{k}$ 。 $\mu_{k}=\pi$. We define the embedding $\hat{\mu}: D \rightarrow \widehat{D}$ by

$$
\hat{\mu}=\lim _{k} \mu_{k} \text {. }
$$

Proposition 1.1. (i) $\hat{\mu}(D)$ is a connected component of $\widehat{D}$. $\hat{\mu}(D)$ is dense in $\widehat{D}$.

Proof. (i) Let

$$
\hat{d}_{0}=\lim d_{0 k} \in \hat{\mu}(D) \subset \widehat{D} .
$$

If

$$
\hat{d}_{1}=\lim _{1 k} \in \widehat{D}
$$

is in the connected component of $\widehat{D}$ containing $\hat{d}_{0}$, then there is a continuous map $\hat{c}:[0,1] \rightarrow \widehat{D}$ such that $\hat{c}(0)=\hat{d}_{0}$ and $\hat{c}(1)=\hat{d}_{1}$. Then $\hat{c}$ can be represented by

$$
\lim c_{k},
$$

where each $c_{k}$ is a continuous map from $[0,1]$ to $X_{k}$ such that

$$
\pi_{j k} \circ c_{j}=c_{k} \text { for } j<k
$$


here the maps $\pi_{j k}: X_{j} \rightarrow X_{k}$ are the natural projections. If $c:[0,1] \rightarrow$ $D$ is the common lifting of the maps $c_{k}$, then $\hat{\mu}(c(0))=\hat{d}_{0}$ and

$$
\hat{\mu}(c(1))=\hat{d}_{1} \in \hat{\mu}(D) .
$$

Thus $\hat{\mu}(D)$ is a connected component of $\widehat{D}$.

(ii) Let

$$
\hat{x}=\lim _{\longleftarrow} x_{k} \in \hat{D},
$$

and let $N\left(\hat{x},\left\{U_{k}\right\}\right)$ be the neighborhood of $\hat{x}$ associated to a collection $\left\{U_{k}\right\}$ of open sets with $x_{k} \in U_{k} \subset X_{k}$ for each $k$. If $x_{0}$ is an element of $X$ with $\pi_{k}\left(x_{k}\right)=x_{0}$ for all $k$, we choose $y_{k} \in \pi_{k}^{-1}\left(x_{0}\right) \cap U_{k}$ for each $k$. If $y \in D$ is an element with $\mu_{k}(y)=y_{k}$ for all $k$, then $\hat{\mu}(y) \in N\left(\hat{x},\left\{U_{k}\right\}\right)$. Thus $\hat{\mu}(D)$ is dense in $\widehat{D}$.

Let $\Gamma(X) \subset \operatorname{Aut}(D)$ be the fundamental group of $X$ and let

$$
\begin{array}{r}
G_{a}(X)=\left\{g \in \operatorname{Aut}(D) \mid\left[\Gamma(X): g \Gamma(X) g^{-1} \cap \Gamma(X)\right]<\infty\right. \\
\text { and } \left.\left[\Gamma(X): g^{-1} \Gamma(X) g \cap \Gamma(X)\right]<\infty\right\} .
\end{array}
$$

The natural homomorphism $G \rightarrow \operatorname{Aut}(D)$ induces the homomorphism

$$
\alpha: \mathbf{G}(\mathbf{Q}) \rightarrow \operatorname{Aut}(D) .
$$

Since $\mathbf{G}$ has no factors defined over $\mathbf{Q}$ and compact over $\mathbf{R}$, the kernel of $\alpha$ is the center $Z_{\mathbf{Q}}$ of $\mathbf{G}(\mathbf{Q})$ and therefore it is finite. The image of $\Gamma \subset \mathbf{G}(\mathbf{Q})$ under $\alpha$ coincides with $\Gamma(X)$. If $\hat{\pi}: \widehat{D} \rightarrow X$ denotes the natural projection, we set

$$
\widehat{G}_{a}(X)=\operatorname{Aut}(\widehat{D}), \quad \widehat{\Gamma}(X)=\left\{\hat{g} \in \widehat{G}_{a}(X) \mid \hat{\pi} \circ \hat{g}=\hat{\pi}\right\} .
$$

To define a homomorphism $\hat{\chi}: G_{a}(X) \rightarrow \widehat{G}_{a}(X)$, we take an element $g \in G_{a}(X)$. For each $k, g$ induces a map $\hat{\chi}_{k}(g): \widehat{D} \rightarrow X_{k}$ (see [6, p. 158]). We set

$$
\hat{\chi}(g)=\lim _{\hat{\chi}_{k}}(g) \in \operatorname{Aut}(\widehat{D}) .
$$

Then we have

$$
\hat{\mu}(g d)=\hat{\chi}(g) \hat{\mu}(d) \quad \text { for } d \in D \text { and } g \in G_{a}(X) .
$$

Since $\hat{\mu}(D)$ is dense in $\widehat{D}$, we have

$$
\hat{\chi}\left(g_{1} g_{2}\right)=\hat{\chi}\left(g_{1}\right) \hat{\chi}\left(g_{2}\right) \text { for } g_{1}, g_{2} \in G_{a}(X) ;
$$

hence $\hat{\chi}: G_{a}(X) \rightarrow \widehat{G}_{a}(X)$ is a homomorphism. 
Proposition 1.2. Let $\hat{d}_{0}$ be an element of $\hat{\mu}(D)$. Then $\hat{\chi}$ is an isomorphism between $G_{a}(X)$ and the subgroup of $\widehat{G}_{a}(X)$ consisting of all $\hat{g} \in \widehat{G}_{a}(X)$ such that $\hat{d}_{0} \hat{g} \in \hat{\mu}(D)$.

\section{Proof. See [6, Lemma 4].}

The group $\widehat{G}_{a}(X)$ is a complete locally compact topological group relative to the topology in which a basis of neighborhoods of the identity consists of the subgroups of finite index in $\widehat{\Gamma}(X)$.

Proposition 1.3. (i) $G_{a}(X)$ is dense in $\widehat{G}_{a}(X)$.

(ii) $\hat{\chi}$ induces an isomorphism between the double cosets $\Gamma(X) \backslash G_{a}(X) / \Gamma(X)$ and $\widehat{\Gamma}(X) \backslash \widehat{G}_{a}(X) / \widehat{\Gamma}(X)$.

\section{Proof. (i) See [6, Theorem 1].}

(ii) This follows from [6, Lemma 3 and Lemma $\left.3^{\prime}\right]$.

Let $\sigma \in \operatorname{Aut}(\mathbf{C})$. Then we consider the complex variety $X^{\sigma}$ obtained from $X$ by the base change. Let $D^{\sigma}$ be the universal covering manifold of $X^{\sigma}$, and let

$$
\Gamma^{\sigma}=\Gamma\left(X^{\sigma}\right) \subset \operatorname{Aut}\left(D^{\sigma}\right)
$$

be the fundamental group of $X^{\sigma}$. If the varieties $X_{k}^{\sigma}$ are the conjugates of $X_{k}$, we set

$$
\widehat{D}^{\sigma}=\lim _{k} X_{k}^{\sigma}, \quad \widehat{G}_{a}\left(X^{\sigma}\right)=\operatorname{Aut}\left(\widehat{D}^{\sigma}\right) .
$$

Then $\widehat{G}_{a}\left(X^{\sigma}\right)$ is a complete locally compact topological group in the topology of subgroups of finite index in

$$
\widehat{\Gamma}\left(X^{\sigma}\right)=\left\{\hat{g}^{\sigma} \in \widehat{G}_{a}\left(X^{\sigma}\right) \mid \hat{\pi}^{\sigma} \circ \hat{g}^{\sigma}=\hat{\pi}^{\sigma}\right\},
$$

where $\hat{\Gamma}^{\sigma}: \widehat{D}^{\sigma} \rightarrow X^{\sigma}$ is the natural projection. As in the case of $X$, we can construct the homomorphism $\hat{\chi}^{\sigma}: G_{a}\left(X^{\sigma}\right) \rightarrow \widehat{G}_{a}\left(X^{\sigma}\right)$, where

$$
\begin{array}{r}
G_{a}\left(X^{\sigma}\right)=\left\{g^{\sigma} \in \operatorname{Aut}\left(D^{\sigma}\right) \mid\left[\Gamma^{\sigma}: g^{\sigma} \Gamma^{\sigma}\left(g^{\sigma}\right)^{-1} \cap \Gamma^{\sigma}\right]<\infty\right. \\
\text { and } \left.\left[\Gamma^{\sigma}:\left(g^{\sigma}\right)^{-1} \Gamma^{\sigma} g^{\sigma} \cap \Gamma^{\sigma}\right]<\infty\right\} .
\end{array}
$$

Let $V \subset G_{a}(X)$ be a subgroup with $[\Gamma(X): V \cap \Gamma(X)]<\infty$, and let $\widehat{V}$ be the closure of $\hat{\chi}(V)$ in $\widehat{G}_{a}(X)$. If $\hat{g} \in \widehat{V} \subset \widehat{G}_{a}(X)$, then there are morphisms $g_{k}: Z_{k} \rightarrow X_{k}$ such that

$$
\lim Z_{k}=\widehat{D}=\lim _{\longleftarrow} X_{k}, \quad \hat{g}=\lim g_{k} .
$$


Applying $\sigma$ to $g_{k}$, we obtain morphisms $g_{k}^{\sigma}: Z_{k}^{\sigma} \rightarrow X_{k}^{\sigma}$. Then

$$
\hat{g}^{\sigma}=\lim _{k}^{\sigma}
$$

is an element of $\widehat{G}\left(X^{\sigma}\right)=\operatorname{Aut}\left(\widehat{D}^{\sigma}\right)$. We set $\widehat{V}^{\sigma}=\left\{\hat{g}^{\sigma} \mid \hat{g} \in \widehat{V}\right\}$ and define the subgroup $V^{\sigma}$ of $G_{a}\left(X^{\sigma}\right)$ by

$$
V^{\sigma}=\left(\hat{\chi}^{\sigma}\right)^{-1}\left(\widehat{V}^{\sigma} \cap \operatorname{Im}\left(\hat{\chi}^{\sigma}\right)\right) .
$$

Now let $G_{a}$ be a subgroup of finite index in $\alpha(\mathbf{G}(\mathbf{Q}))$ containing $\alpha(\Gamma)=\Gamma(X)$, and let

$$
G_{a}^{\sigma}=\left(G_{a}\right)^{\sigma} \subset G_{a}\left(X^{\sigma}\right)
$$

Proposition 1.4. $G_{a}^{\sigma}$ is dense in the connected component of the identity of $\operatorname{Aut}\left(D^{\sigma}\right)$ in the ordinary topology.

Proof. $G_{a}^{\sigma}$ is contained in the connected component of the identity of $\operatorname{Aut}\left(D^{\sigma}\right)$ by [10, Lemma 3.7]. The density follows from [6, Theorem 5] (see also [6, Theorem A.7]).

THEOREM 1.5. The group $\Gamma^{\sigma}=\Gamma\left(X^{\sigma}\right)$ is an arithmetic subgroup of the connected component of the identity of $\operatorname{Aut}\left(D^{\sigma}\right)$.

Proof. This follows from the main theorems in [6] and [7].

2. The homomorphism $\hat{\rho}$. Let $\mathbf{G}, G, K, D, \Gamma$ and $X$ be as in $\S 1$. We consider another semisimple algebraic $\mathbf{Q}$-group $\mathbf{G}^{\prime}$ and its arithmetic subgroup $\Gamma^{\prime} \subset \mathbf{G}^{\prime}(\mathbf{Q})$ that is torsion free. As in the case of $\mathbf{G}$, we associate $G^{\prime}, K^{\prime}, D^{\prime}$ and the arithmetic variety $X^{\prime}=\Gamma^{\prime} \backslash D^{\prime}$ to $\mathbf{G}^{\prime}$. Let $\rho: G \rightarrow G^{\prime}$ be a homomorphism, $\phi: X \rightarrow X^{\prime}$ a morphism of varieties, and $\tau: D \rightarrow D^{\prime}$ a holomorphic lifting of $\phi$ such that $\rho$ and $\tau$ are equivariant, i.e.,

$$
\tau(g y)=\rho(g) \tau(y) \text { for all } g \in G \text { and } y \in D .
$$

Let $\left\{\Gamma_{k}\right\},\left\{X_{k}\right\}$ and $\widehat{D}$ be as in $\S 1$, and let $\left\{\Gamma_{k}^{\prime}\right\}$ be an inductive system of arithmetic subgroups of finite index of $\Gamma^{\prime}$ such that $\rho\left(\Gamma_{k}\right) \subset \Gamma_{k}^{\prime}$ for each $k \geq 1$. The quotient spaces $X_{k}^{\prime}=\Gamma_{k}^{\prime} \backslash D^{\prime}$ are arithmetic varieties and they form a projective system $\left\{X_{k}^{\prime}\right\}$ of covering manifolds of $X^{\prime}$. The holomorphic map $\tau: D \rightarrow D^{\prime}$ induces a 
morphism $\phi: X_{k} \rightarrow X_{k}^{\prime}$ for each $k \geq 1$. We set

$$
\widehat{D}^{\prime}=\varliminf_{k} X_{k}^{\prime}, \quad \widehat{G}_{a}\left(X^{\prime}\right)=\operatorname{Aut}\left(\widehat{D}^{\prime}\right),
$$

and define the holomorphic map $\hat{\tau}: \widehat{D} \rightarrow \widehat{D}^{\prime}$ by

$$
\hat{\tau}=\varliminf_{\longleftarrow} \phi_{k} .
$$

For each $\hat{g} \in \widehat{G}_{a} \subset \widehat{G}_{a}(X)=\operatorname{Aut}(\widehat{D})$, there are elements

$$
g_{k} \in \alpha^{-1}\left(G_{a}\right) \subset \mathbf{G}(\mathbf{Q})
$$

(recall that $\operatorname{Ker}(\alpha)=Z_{\mathbf{Q}}$ is finite) such that

$$
\hat{g}=\lim _{\longleftarrow} p_{k}\left(g_{k}\right),
$$

where the morphisms $p_{k}\left(g_{k}\right)$ are determined as follows: Let

$$
\begin{aligned}
\Gamma_{g, k} & =\Gamma_{k} \cap g_{k}^{-1} \Gamma_{k} g_{k}, \\
X_{g, k} & =\Gamma_{g, k} \backslash D .
\end{aligned}
$$

Then, for each $k$, we define

$$
p_{k}\left(g_{k}\right): X_{g, k} \rightarrow X_{k}
$$

to be the morphism of arithmetic varieties induced by the left multiplication $g_{k}: D \rightarrow D, D=G / K$. We have

$$
g_{k}=\gamma_{j, k} g_{j} \text { with } \gamma_{j, k} \in \Gamma_{j} \text { for } j \leq k .
$$

Recall that $\left\{\Gamma_{k}^{\prime}\right\}$ is an inductive system of arithmetic subgroups of finite index of $\Gamma^{\prime}$ such that $\rho\left(\Gamma_{k}\right) \subset \Gamma_{k}^{\prime}$ for each $k$. We set

$$
\begin{aligned}
\Gamma_{g, k}^{\prime} & =\Gamma_{k}^{\prime} \cap \rho\left(g_{k}\right)^{-1} \Gamma_{k}^{\prime} \rho\left(g_{k}\right), \\
X_{g, k}^{\prime} & =\Gamma_{g, k}^{\prime} \backslash D^{\prime}
\end{aligned}
$$

for each $k$. Then we have $\rho\left(\Gamma_{g, k}\right) \subset \Gamma_{g, k}^{\prime}$, and hence the left multiplication maps $\rho\left(g_{k}\right): D^{\prime} \rightarrow D^{\prime}$ induce the morphisms

$$
p_{k}^{\prime}\left(\rho g_{k}\right): X_{g, k}^{\prime} \rightarrow X_{k}^{\prime} \text {. }
$$

Since we have

$$
\rho\left(g_{k}\right)=\rho\left(\gamma_{j, k} g_{j}\right)=\rho\left(\gamma_{j, k}\right) \rho\left(g_{j}\right)
$$

with

$$
\rho\left(\gamma_{j, k}\right) \in \rho\left(\Gamma_{j}\right) \subset \Gamma_{j}^{\prime} \text { for } j \leq k,
$$


the collection $\left\{\rho\left(g_{k}\right)\right\}$ of liftings $\rho\left(g_{k}\right)$ of $p_{k}^{\prime}\left(\rho g_{k}\right)$ determines the projective limit

$$
\lim _{k} p_{k}^{\prime}\left(\rho g_{k}\right) \in \widehat{G}_{a}\left(X^{\prime}\right)=\operatorname{Aut}\left(\widehat{D}^{\prime}\right) \text {. }
$$

Thus we obtain a homomorphism $\hat{\rho}: \widehat{G}_{a} \rightarrow \widehat{G}_{a}^{\prime}$ defined by

$$
\hat{\rho}(\hat{g})=\lim _{k}^{\prime}\left(\rho g_{k}\right)
$$

for all $\hat{g}=\lim g_{k} \in \widehat{G}_{a}$.

Proposition 2.1. If $\hat{\tau}: \widehat{D} \rightarrow \widehat{D}^{\prime}$ and $\hat{\rho}: \widehat{G}_{a} \rightarrow \widehat{G}_{a}\left(X^{\prime}\right)$ are as described above, then

$$
\hat{\tau}(\hat{g} \hat{y})=\hat{\rho}(\hat{g}) \hat{\tau}(\hat{y}) \quad \text { for all } \hat{g} \in \widehat{G}_{a} \text { and } \hat{y} \in \hat{D} .
$$

Proof. If $\mu_{k}: D \rightarrow X_{k}, \mu_{g, k}: D \rightarrow X_{g, k}, \mu_{k}^{\prime}: D^{\prime} \rightarrow X_{k}^{\prime}$ and $\mu_{g, k}^{\prime}: D^{\prime} \rightarrow X_{g, k}^{\prime}$ are the natural covering maps, then we have

$$
\begin{aligned}
\mu_{k}^{\prime} \tau\left(g_{k} y\right) & =\phi_{k}\left(g_{k} \mu_{g, k}(y)\right), \\
\mu_{k}^{\prime} \rho\left(g_{k}\right) \tau(y) & =\left(p^{\prime}\left(\rho g_{k}\right)\right) \phi_{g, k}\left(\mu_{g, k}(y)\right)
\end{aligned}
$$

for all $y \in D$, where

$$
\phi_{g, k}: X_{g, k} \rightarrow X_{g, k}^{\prime}
$$

is the morphism induced by $\tau: D \rightarrow D$ for each $k$ with

$$
\tau=\lim \phi_{g, k} \text {. }
$$

Since $\tau\left(g_{k} y\right)=\rho\left(g_{k}\right) \tau(y)$ for all $y \in D$, we have

$$
\phi_{k}\left(g_{k} \mu_{g, k}(y)\right)=\left(p_{k}^{\prime}\left(\rho g_{k}\right)\right) \phi_{g, k}\left(\mu_{g, k}(y)\right)
$$

for all $y \in D$. Since $\mu_{g, k}: D \rightarrow X_{g, k}$ is surjective and

$$
\hat{\rho}(\hat{g})=\lim _{\longleftarrow} p_{k}^{\prime}\left(\rho g_{k}\right),
$$

it follows that

$$
\hat{\tau}(\hat{g} \hat{y})=\hat{\rho}(\hat{g}) \hat{\tau}(\hat{y}) \quad \text { for all } \hat{y}=\lim _{\longleftarrow}\left(\mu_{g, k}(y)\right) \in \hat{D} .
$$

3. Conjugates of $\hat{\rho}$. Let $\left\{X_{k}\right\}$ and $\left\{X_{k}^{\prime}\right\}$ be the projective systems of finite unramified covering manifolds of $X$ and $X^{\prime}$, respectively, considered in $\S 2$. We fix an element $\sigma \in \operatorname{Aut}(\mathbf{C})$. The varieties $X^{\sigma}, X^{\prime \sigma}, X_{k}^{\sigma}$ and $X_{k}^{\prime \sigma}$ are arithmetic varieties, and the collections 
$\left\{X_{k}^{\sigma}\right\}$ and $\left\{X_{k}^{\prime \sigma}\right\}$ are projective systems of finite unramified covering manifolds of $X^{\sigma}$ and $X^{\prime \sigma}$ respectively (see [5], [6], [7], [10]). Let $\phi_{k}^{\sigma}: X_{k}^{\sigma} \rightarrow X_{k}^{\prime \sigma}$ be the conjugate morphism of $\phi_{k}: X_{k} \rightarrow X_{k}^{\prime}$ for each $k$. Let $\widehat{D}, \widehat{G}_{a}\left(X^{\sigma}\right)$ and $\widehat{\Gamma}\left(X^{\sigma}\right)$ be as in $\S 1$, and let

$$
\begin{aligned}
\widehat{D}^{\prime \sigma} & =\lim _{k} X_{k}^{\prime \sigma}, \quad \widehat{G}_{a}\left(X^{\prime \sigma}\right)=\operatorname{Aut}\left(\widehat{D}^{\prime \sigma}\right), \\
\widehat{\Gamma}\left(X^{\prime \sigma}\right) & =\left\{\hat{g}^{\prime \sigma} \in \widehat{G}_{a}\left(X^{\prime \sigma}\right) \mid \hat{\pi}^{\prime \sigma} \circ \hat{g}^{\prime \sigma}=\hat{\pi}^{\prime \sigma}\right\},
\end{aligned}
$$

where $\hat{\pi}^{\prime \sigma}: \widehat{D}^{\prime \sigma} \rightarrow X^{\prime \sigma}$ is the natural projection. We define the map $\hat{\tau}^{\sigma}: \widehat{D}^{\sigma} \rightarrow \widehat{D}^{\prime \sigma}$ by

$$
\hat{\tau}^{\sigma}=\lim _{k}^{\sigma} .
$$

If $\hat{h} \in \widehat{G}_{a}^{\sigma}$, then there exist a projective system $\left\{Z_{k}\right\}$ of finite unramified covering manifolds of $X^{\sigma}$ and morphisms $h_{k}: Z_{k} \rightarrow X_{k}^{\sigma}$ such that

$$
\stackrel{\lim }{\longleftarrow} Z_{k}=\widehat{D}^{\sigma}=\lim _{k} X_{k}^{\sigma}, \quad \hat{h}=\lim ^{\lim } h_{k} .
$$

The morphisms $h_{k}$ induce the morphisms

$$
h_{k}^{\sigma^{-1}}: Z_{k}^{\sigma^{-1}} \rightarrow X_{k}
$$

Let $\tilde{h}_{k}^{\sigma^{-1}}: D \rightarrow D$ be an element of $\alpha^{-1}\left(G_{a}(X)\right) \subset \mathbf{G}(\mathbf{Q})$ that is a lifting of $h_{k}^{\sigma^{-1}}$ for each $k$. We define the homomorphism $\hat{\rho}^{\sigma}: \widehat{G}_{a}^{\sigma} \rightarrow$ $\widehat{G}_{a}\left(X^{\prime \sigma}\right)$ by

$$
\hat{\rho}^{\sigma}(\hat{h})=\lim _{(}\left(p_{k}^{\prime}\left(\rho\left(\hat{h}_{k}^{\sigma^{-1}}\right)\right)\right)^{\sigma}
$$

where

$$
p_{k}^{\prime}\left(\rho\left(\tilde{h}_{k}^{\sigma^{-1}}\right)\right): W_{k}^{\prime} \rightarrow X_{k}^{\prime}
$$

is the morphism induced from $\rho\left(\tilde{h}_{k}^{\sigma^{-1}}\right): D^{\prime} \rightarrow D^{\prime}$ for each $k$.

PROPOsItion 3.1. $\hat{\tau}^{\sigma}\left(\hat{g}^{\sigma} \hat{y}^{\sigma}\right)=\hat{\rho}^{\sigma}\left(\hat{g}^{\sigma}\right) \hat{\tau}^{\sigma}\left(\hat{y}^{\sigma}\right)$ for all $\hat{g}^{\sigma} \in \widehat{G}_{a}^{\sigma}$ and $\hat{y}^{\sigma} \in \widehat{D}^{\sigma}$.

Proof. For each $k$ the relation $\hat{\tau}(\hat{g} \hat{y})=\hat{\rho}(\hat{g}) \hat{\tau}(\hat{y})$ induces the following commutative diagram:

$$
\begin{aligned}
X_{g, k} & \stackrel{\phi_{g, k}}{\longrightarrow} \\
p_{k}\left(g_{k}\right) \downarrow & X_{g, k}^{\prime} \\
X_{k} \stackrel{\phi_{k}}{\longrightarrow} X_{k}^{\prime} & X_{k}^{\prime}\left(\rho g_{k}\right)
\end{aligned}
$$


Applying $\sigma$ to this diagram, we obtain

$$
\begin{aligned}
X_{g, k}^{\sigma} & \stackrel{\phi_{g, k}^{\sigma}}{\longrightarrow} X_{g, k}^{\prime \sigma} \\
\left(p_{k}\left(g_{k}\right)\right)^{\sigma} \downarrow & \\
X_{k}^{\sigma} & \stackrel{\phi_{k}^{\sigma}}{\longrightarrow} X_{k}^{\prime \sigma}
\end{aligned}
$$

so that

$$
\phi_{k}^{\sigma}\left(\left(p_{k}\left(g_{k}\right)\right)^{\sigma} x_{k}^{\sigma}\right)=\left(p_{k}^{\prime}\left(\rho g_{k}\right)\right)^{\sigma} \phi_{g, k}^{\sigma}\left(x_{k}^{\sigma}\right)
$$

for all $x_{k}^{\sigma} \in X_{g, k}^{\sigma}$. By taking projective limits we obtain

$$
\hat{\tau}^{\sigma}\left(\hat{g}^{\sigma} \hat{y}^{\sigma}\right)=\hat{\rho}^{\sigma}\left(\hat{g}^{\sigma}\right) \hat{\tau}^{\sigma}\left(\hat{y}^{\sigma}\right)
$$

for all $\hat{y}^{\sigma} \in \widehat{D}^{\sigma}$.

Proposition 3.2. $\hat{\rho}\left(\widehat{\Gamma}\left(X^{\sigma}\right)\right)$ is contained in $\widehat{\Gamma}\left(X^{\prime \sigma}\right)$.

Proof. If $\hat{h} \in \widehat{\Gamma}\left(X^{\sigma}\right) \subset \widehat{G}_{a}\left(X^{\sigma}\right)$, there are morphisms $h_{k}: Z_{k} \rightarrow X_{k}^{\sigma}$ such that

$$
\stackrel{\lim }{\longleftarrow} Z_{k}=\widehat{D}^{\sigma}=\lim _{k} X_{k}^{\sigma}, \quad \hat{h}=\lim _{k} h_{k} \quad \text { and } \pi_{k}^{\sigma} \circ h_{k}=\pi_{k}^{0},
$$

where $\pi_{k}^{0}: Z_{k} \rightarrow X^{\sigma}$ is the natural projection. Applying $\sigma^{-1}$, we obtain the morphisms $h_{k}^{\sigma^{-1}}: Z_{k}^{\sigma^{-1}} \rightarrow X_{k}$ such that

$$
\pi_{k} \circ h_{k}^{\sigma^{-1}}=\left(\pi_{k}^{0}\right)^{\sigma^{-1}} \text { for all } k,
$$

where the maps $\left(\pi_{k}^{0}\right)^{\sigma^{-1}}: Z_{k} \rightarrow X$ are the natural projections. If the maps $\tilde{h}_{k}^{\sigma^{-1}}: D \rightarrow D$ with $\tilde{h}_{k}^{\sigma^{-1}} \in \mathbf{G}(\mathbf{Q})$ are as before, we have $\tilde{h}_{k}^{\sigma^{-1}}(\Gamma) \subset \Gamma$, and therefore

$$
\rho\left(\tilde{h}_{k}^{\sigma^{-1}}\right)\left(\Gamma^{\prime}\right) \subset \Gamma^{\prime} \quad \text { for all } k .
$$

Thus the morphisms $p_{k}^{\prime}\left(\rho\left(\tilde{h}_{k}^{\sigma^{-1}}\right)\right): W_{k}^{\prime} \rightarrow X_{k}^{\prime}$ satisfy

$$
\pi_{k}^{\prime 0} \circ p_{k}^{\prime}\left(\rho\left(\tilde{h}_{k}^{\sigma^{-1}}\right)\right)=\pi_{k}^{\prime} \text { for all } k
$$

where the maps $\pi_{k}^{\prime 0}: W_{k}^{\prime} \rightarrow X^{\prime}$ are the natural projections. Hence we have

$$
\left(\pi_{k}^{\prime 0}\right)^{\sigma} \circ\left(p_{k}^{\prime}\left(\rho\left(\tilde{h}_{k}^{\sigma^{-1}}\right)\right)\right)^{\sigma}=\left(\pi_{k}^{\prime 0}\right)^{\sigma}
$$

for all $k$. Thus it follows that $\hat{\rho}^{\sigma}(\hat{h}) \in \widehat{\Gamma}\left(X^{\prime \sigma}\right)$. 
4. The homomorphism $\rho^{\sigma}$. Let $X^{\sigma}, D^{\sigma}, \Gamma^{\sigma}, G_{a}^{\sigma}, G_{a}\left(X^{\sigma}\right), \widehat{D}^{\sigma}$, $\widehat{\Gamma}\left(X^{\sigma}\right), \widehat{G}_{a}\left(X^{\sigma}\right)$ be as in $\S 1$, and let $\widehat{D}^{\prime \sigma}, \widehat{\Gamma}_{a}\left(X^{\prime \sigma}\right), \widehat{G}\left(X^{\prime \sigma}\right)$ be as in $\S 3$. Let $D^{\prime \sigma}$ be the universal covering space of $X^{\prime \sigma}$, and let

$$
\Gamma^{\sigma}=\Gamma\left(X^{\prime \sigma}\right) \subset \operatorname{Aut}\left(D^{\prime \sigma}\right)
$$

be the fundamental group of $X^{\prime \sigma}$. We set

$$
\begin{array}{r}
G_{a}\left(X^{\prime \sigma}\right)=\left\{g^{\prime \sigma} \in \operatorname{Aut}\left(D^{\prime \sigma}\right) \mid\left[\Gamma^{\prime \sigma}: g^{\prime \sigma} \Gamma^{\prime \sigma}\left(g^{\prime \sigma}\right)^{-1} \cap \Gamma^{\prime \sigma}\right]<\infty\right. \\
\text { and } \left.\left[\Gamma^{\prime \sigma}:\left(g^{\prime \sigma}\right)^{-1} \Gamma^{\prime \sigma} g^{\prime \sigma} \cap \Gamma^{\prime \sigma}\right]<\infty\right\} .
\end{array}
$$

Since the map $\hat{\mu}: D \rightarrow \widehat{D}$ and the homomorphism $\hat{\chi}: G_{a}(X) \rightarrow \widehat{G}_{a}(X)$ described in $\S 1$ are injective, we shall identify each of $D^{\sigma}, D^{\prime \sigma}$, $G_{a}\left(X^{\sigma}\right), G_{a}\left(X^{\prime \sigma}\right)$ with its embedded image in $\widehat{D}^{\sigma}, \widehat{D}^{\prime \sigma}, \widehat{G}_{a}\left(X^{\sigma}\right)$, $\widehat{G}_{a}\left(X^{\prime \sigma}\right)$ respectively. By Proposition 1.1(i) and the above identification, $D^{\sigma}$ and $D^{\prime \sigma}$ are connected components of $\widehat{D}^{\sigma}$ and $\widehat{D}^{\prime \sigma}$ respectively. We shall assume that $D^{\prime \sigma}$ is the connected component of $\widehat{D}^{\prime \sigma}$ containing the image of $D^{\sigma}$ under the map $\tau^{\sigma}$.

Proposition 4.1. If $D^{\prime \sigma}$ is the connected component of $\widehat{D}^{\prime \sigma}$ chosen as above, then $\hat{\rho}^{\sigma}\left(G_{a}^{\sigma}\right)$ is contained in $G_{a}\left(X^{\prime \sigma}\right)$.

Proof. Let $d_{0}^{\sigma} \in D^{\sigma} \subset \widehat{D}^{\sigma}$. Then we have

$$
\hat{\tau}^{\sigma}\left(d_{0}^{\sigma}\right) \in D^{\prime \sigma} \subset \widehat{D}^{\prime \sigma} .
$$

By Proposition 1.2 we have

$$
\begin{aligned}
& G_{a}^{\sigma} \subset G_{a}\left(X^{\sigma}\right)=\left\{\hat{g}^{\sigma} \in \widehat{G}_{a}\left(X^{\sigma}\right) \mid \hat{g}^{\sigma} d_{0}^{\sigma} \in D^{\sigma}\right\}, \\
& G_{a}\left(X^{\prime \sigma}\right)=\left\{\hat{g}^{\prime \sigma} \in \widehat{G}_{a}\left(X^{\prime \sigma}\right) \mid \hat{g}^{\prime \sigma} \hat{\tau}^{\sigma}\left(d_{0}^{\sigma}\right) \in D^{\prime \sigma}\right\} .
\end{aligned}
$$

If $g^{\sigma} \in G_{a}^{\sigma}$, then $g^{\sigma} d_{0}^{\sigma} \in D^{\sigma}$; hence from the continuity of $\hat{\tau}^{\sigma}$ it follows that

$$
\hat{\tau}^{\sigma}\left(g^{\sigma} d_{0}^{\sigma}\right) \in \hat{\tau}^{\sigma}\left(D^{\sigma}\right) \subset D^{\prime \sigma} .
$$

Thus we get

$$
\hat{\rho}^{\sigma}\left(g^{\sigma}\right) \hat{\tau}^{\sigma}\left(d_{0}^{\sigma}\right)=\hat{\tau}^{\sigma}\left(g^{\sigma} d_{0}^{\sigma}\right) \in D^{\prime \sigma} .
$$

Therefore it follows that

$$
\hat{\rho}^{\sigma}\left(g^{\sigma}\right) \in G_{a}\left(X^{\prime \sigma}\right) \text { for all } g^{\sigma} \in G_{a}^{\sigma} .
$$

Now we define the homomorphism $\rho^{\sigma}: G_{a}^{\sigma} \rightarrow G_{a}\left(X^{\prime \sigma}\right)$ and $\tau^{\sigma}$ : $D^{\sigma} \rightarrow D^{\prime \sigma}$ by

$$
\rho^{\sigma}=\left.\hat{\rho}^{\sigma}\right|_{G_{a}^{\sigma}}, \quad \tau^{\sigma}=\left.\hat{\tau}^{\sigma}\right|_{D^{\sigma}} .
$$


Then $\rho^{\sigma}$ and $\tau^{\sigma}$ satisfy the relation

$$
\tau^{\sigma}\left(g^{\sigma} d^{\sigma}\right)=\rho^{\sigma}\left(g^{\sigma}\right) \tau^{\sigma}\left(d^{\sigma}\right)
$$

for all $g^{\sigma} \in G_{a}^{\sigma}$ and $d^{\sigma} \in D^{\sigma}$.

5. Conjugates of equivariant pairs. Let $\rho: G \rightarrow G^{\prime}$ and $\tau: D \rightarrow D^{\prime}$ be equivariant as before so that we have

$$
\tau(g z)=\rho(g) \tau(z)
$$

for all $g \in G$ and $z \in D$. Also as before let $\Gamma \subset G$ and $\Gamma^{\prime} \subset G^{\prime}$ be torsion free arithmetic subgroups with $\rho(\Gamma) \subset \Gamma^{\prime}$, and let $\phi: X \rightarrow X^{\prime}$ be the morphism induced by $\tau$ where $X=\Gamma \backslash D$ and $X^{\prime}=\Gamma^{\prime} \backslash$ $D^{\prime}$. If $\rho^{\sigma}: G_{a}^{\sigma} \rightarrow G_{a}^{\prime \sigma}$ and $\tau^{\sigma}: D^{\sigma} \rightarrow D^{\prime \sigma}$ are as in $\S 4$, we denote by $G_{0}^{\sigma}, G_{0}^{\prime \sigma}$ the connected components of the identity of $\operatorname{Aut}\left(D^{\sigma}\right)$, $\operatorname{Aut}\left(D^{\prime \sigma}\right)$ respectively. By Proposition $1.4 G_{a}^{\sigma}$ is dense in $G_{0}^{\sigma}$; hence by Proposition 3.1 and Proposition 4.1, we have

$$
\tau^{\sigma}(h y)=\rho^{\sigma}(h) \tau^{\sigma}(y)
$$

for all $h \in G_{a}^{0}$ and $y \in D^{\sigma}$. We set

$$
H=\left\{\left(g, g^{\prime}\right) \in G_{0}^{\sigma} \times G_{0}^{\sigma} \mid \tau^{\sigma}(g y)=g^{\prime} \tau^{\sigma}(y) \text { for all } y \in D^{\sigma}\right\} .
$$

Since the set $\left\{\left(g, \rho^{\sigma}(g)\right) \mid g \in G_{a}^{\sigma}\right\}$ is contained in $H$ and $G_{a}^{\sigma}$ is dense in $G_{0}^{\sigma}$, the projection map $\operatorname{pr}_{1}: H \rightarrow G_{0}^{\sigma}$ is surjective.

Proposition 5.1. $H$ is a reductive Lie group.

Proof. Let $K$ be the kernel of the projection map $\mathrm{pr}_{1}: H \rightarrow G_{0}^{\sigma}$. Then we have

$$
\begin{aligned}
K & =\left\{\left(1, g^{\prime}\right) \in G_{0}^{\sigma} \times G_{0}^{\prime \sigma} \mid \tau^{\sigma}(y)=g^{\prime} \tau^{\sigma}(y) \text { for all } y \in D^{\sigma}\right\} \\
& \cong\left\{g^{\prime} \in G_{0}^{\prime \sigma} \mid \tau^{\sigma}(y)=g^{\prime} \tau^{\sigma}(y) \text { for all } y \in D^{\sigma}\right\}=\bigcap_{y \in D^{\sigma}} \operatorname{Iso}\left(\tau^{\sigma}(y)\right),
\end{aligned}
$$

where $\operatorname{Iso}\left(\tau^{\sigma}(y)\right)$ is the isotropy subgroup of $\tau^{\sigma}(y)$ in $G_{0}^{\prime \sigma}$. Hence $K$ is a compact Lie group, and therefore a reductive Lie group. Since there is an exact sequence

$$
1 \rightarrow K \rightarrow H \stackrel{\mathrm{pr}_{1}}{\longrightarrow} G_{0}^{\sigma} \rightarrow 1
$$

of Lie groups with $K$ and $G_{0}^{\sigma}$ reductive, it follows that $H$ is reductive. 
THEOREM 5.2. There exist a finite covering $G_{1}^{\sigma}$ of $G_{0}^{\sigma}$ and a homomorphism $\rho_{1}^{\sigma}: G_{1}^{\sigma} \rightarrow G_{0}^{\prime \sigma}$ of Lie groups such that $\rho_{1}^{\sigma}$ and $\tau^{\sigma}$ are equivariant and $\rho_{1}^{\sigma}\left(\Gamma^{\sigma}\right)$ is contained in $\Gamma^{\sigma}$.

Proof. Decompose the reductive Lie group $H$ into an almost direct product of simple Lie groups and simple tori, and let $G_{1}^{\sigma}$ be the product of the simple factors of $H$ which map nontrivially to $G_{0}^{\sigma}$ (see [2, III.2.2] for a similar argument). Then the kernel of the map from $G_{1}^{\sigma}$ to $G_{0}^{\sigma}$ is finite, and hence $G_{1}^{\sigma}$ is a finite covering Lie group of $G_{0}^{\sigma}$. We define the action of $G_{1}^{\sigma}$ on $D^{\sigma}$ and the homomorphism $\rho_{1}^{\sigma}: G_{1}^{\sigma} \rightarrow G_{0}^{\prime \sigma}$ by

$$
\left(g, g^{\prime}\right) \cdot y=g y, \quad \rho_{1}^{\sigma}\left(g, g^{\prime}\right)=g^{\prime}
$$

for all $y \in D^{\sigma}$ and $\left(g, g^{\prime}\right) \in G_{1}^{\sigma} \subset H \subset G_{0}^{\sigma} \times G_{0}^{\prime \sigma}$. Then we have

$$
\tau^{\sigma}\left(\left(g, g^{\prime}\right) \cdot y\right)=\tau^{\sigma}(g y)=g^{\prime} \tau^{\sigma}(y)=\rho_{1}^{\sigma}\left(g, g^{\prime}\right) \tau^{\sigma}(y)
$$

for all $\left(g, g^{\prime}\right) \in G_{1}^{\sigma}$ and $y \in D^{\sigma}$. Hence we obtain a homomorphism of Lie groups $\rho_{1}^{\sigma}$ from a finite covering $G_{1}^{\sigma}$ of $G_{0}^{\sigma}$ to $G_{0}^{\prime \sigma}$ such that

$$
\tau^{\sigma}\left(h_{1} y\right)=\rho_{1}^{\sigma}\left(h_{1}\right) \tau^{\sigma}(y)
$$

for all $h_{1} \in G_{1}^{\sigma}$ and $y \in D^{\sigma}$. Thus it follows that $\rho_{1}^{\sigma}$ and $\tau^{\sigma}$ are equivariant. Now it remains to show that $\rho_{1}^{\sigma}\left(\Gamma^{\sigma}\right)$ is contained in $\Gamma^{\prime \sigma}$. By Proposition 3.2, $\hat{\rho}\left(\widehat{\Gamma}\left(X^{\sigma}\right)\right)$ is contained in $\widehat{\Gamma}\left(X^{\prime \sigma}\right)$; hence $\hat{\rho}^{\sigma}: \widehat{G}_{a}^{\sigma} \rightarrow \widehat{G}_{a}\left(X^{\prime \sigma}\right)$ induces the mapping

$$
\hat{\rho}^{\sigma}: \widehat{\Gamma}\left(X^{\sigma}\right) \backslash \widehat{G}_{a}^{\sigma} / \widehat{\Gamma}\left(X^{\sigma}\right) \rightarrow \widehat{\Gamma}\left(X^{\prime \sigma}\right) \backslash \widehat{G}_{a}\left(X^{\prime \sigma}\right) / \widehat{\Gamma}\left(X^{\prime \sigma}\right)
$$

of double cosets. By Proposition 1.3(ii) $\hat{\rho}^{\sigma}$ induces the mapping

$$
\hat{\rho}^{\sigma}: \Gamma\left(X^{\sigma}\right) \backslash G_{a}^{\sigma} / \Gamma\left(X^{\sigma}\right) \rightarrow \Gamma\left(X^{\prime \sigma}\right) \backslash G_{a}\left(X^{\prime \sigma}\right) / \Gamma\left(X^{\prime \sigma}\right) .
$$

Thus we have

$$
\hat{\rho}\left(\Gamma\left(X^{\sigma}\right)\right)=\rho^{\sigma}\left(\Gamma^{\sigma}\right) \subset \Gamma\left(X^{\prime \sigma}\right)=\Gamma^{\prime \sigma} .
$$

Since $\rho_{1}^{\sigma}\left(\Gamma^{\sigma}\right)=\rho^{\sigma}\left(\Gamma^{\sigma}\right)$, it follows that $\rho_{1}^{\sigma}\left(\Gamma^{\sigma}\right) \subset \Gamma^{\sigma}$.

6. Conjugates of Kuga fiber varieties. First, we shall describe the construction of Kuga fiber varieties (see [1], [8], [11, Chapter 4] for details). Let $V$ be a Q-vector space of dimension $2 n$, and let $L$ be a lattice in $V$. Let $\beta$ be a nondegenerate alternating bilinear form on $V$ such that $\beta(L, L) \subset \mathbf{Z}$. Let

$$
\operatorname{Sp}(V, \beta)=\{g \in \mathrm{GL}(V) \mid \beta(g x, g y)=\beta(x, y) \text { for all } x, y \in V\}
$$


be the symplectic group of the pair $(V, \beta)$, and let $\mathscr{H}$ denote the Siegel space

$$
\begin{aligned}
\mathscr{H}=\left\{J \in \mathrm{GL}\left(V_{\mathbf{R}}\right) \mid J^{2}=\right. & -1, \beta(x, J y) \text { is a positive definite } \\
& \text { symmetric bilinear form in } \left.x, y \in V_{\mathbf{R}}\right\} .
\end{aligned}
$$

Then each element $J \in \mathscr{H}$ defines a complex structure on $V_{\mathbf{R}}$ and there is a unique complex analytic structure on $\mathscr{H} \times V_{\mathbf{R}}$ such that the projection $P: \mathscr{H} \times V_{\mathbf{R}} \rightarrow \mathscr{H}$ is a complex vector bundle over $\mathscr{H}$. For each $J$ if we denote the complex vector space $\left(V_{\mathbf{R}}, J\right)$ by $V_{J}$, then the complex torus $A_{J}=V_{J} / L$ is an abelian variety with the polarization $\beta$. We set

$$
A_{\mathscr{H}}=L \backslash \mathscr{H} \times V_{\mathbf{R}},
$$

where the action of $L$ on $\mathscr{H} \times V_{\mathbf{R}}$ is given by

$$
l \cdot(J, v)=(J, v+l) \text { for } j \in \mathscr{H} \text { and } l \in L .
$$

Then the vector bundle $P: \mathscr{H} \times V_{\mathbf{R}} \rightarrow \mathscr{H}$ induces the fiber bundle $\pi_{\mathscr{L}}: A_{\mathscr{L}} \rightarrow \mathscr{H}$ whose fibers are abelian varieties polarized by $\beta$. We set

$$
\operatorname{Sp}(L, \beta)=\{g \in \operatorname{Sp}(V, \beta) \mid g L=L\},
$$

and take a subgroup $\Gamma_{S}$ of $\operatorname{Sp}(L, \beta)$ of finite index that contains no elements of finite order. Then, as discussed in $\S 1$, the quotient $X=\Gamma_{S} \backslash \mathscr{H}$ is an arithmetic variety that can be considered as a Zariski open subset of a complex projective variety. Now the fiber bundle $\pi_{\mathscr{H}}: A_{\mathscr{L}} \rightarrow \mathscr{H}$ induces the standard family of abelian varieties $\pi_{S}: Y_{S} \rightarrow X_{S}$ over $X_{S}$.

Let $\mathbf{G}, G$ and $D=G / K$ be as in $\S 1$. Let $\rho: G \rightarrow \operatorname{Sp}(V, \beta)(\mathbf{R})$ be a symplectic representation of $G$, and let $\Gamma$ be a torsion free arithmetic subgroup of $\mathbf{G}$ with $\rho(\Gamma) \subset \Gamma_{S}$. We shall assume that the quotient $X=\Gamma \backslash D$ is compact. Let $\tau: D \rightarrow \mathscr{H}$ be a holomorphic map such that $\rho$ and $\tau$ are equivariant, and let $\phi: X \rightarrow X_{S}$ be the morphism of varieties induced by $\tau$. By pulling back the fiber bundle $\pi_{S}: Y_{S} \rightarrow X_{X}$ via the morphism $\phi: X \rightarrow X_{S}$, we obtain the fiber bundle over the arithmetic variety $X$ whose fibers are abelian varieties polarized by $\beta$. It is known (cf. [8], [11, Theorem 8.6]) that the fiber space $Y$ of the family of abelian varieties $\pi: y \rightarrow X$ has a structure of a complex projective variety. The fiber space $Y$ (or the fiber bundle $\pi: Y \rightarrow X$. itself) is called a Kuga fiber variety.

To consider conjugates of Kuga fiber varieties, we shall first state some of the known results of G. Shimura about the families of abelian varieties associated to PEL-types (see e.g. [12], [15] for details). 
THEOREM 6.1. Let $\Omega$ be a normal admissible PEL-type (see [14] for its definition). Then there exists a family of abelian varieties $f: W \rightarrow$ $U$ denoted by $\mathscr{F}(\Omega)$ with the following properties:

(i) For each $u \in U$, the fiber $f^{-1}(u)$ of $\mathscr{F}(\Omega)$ has a PEL-structure $Q_{u}$ of type $\Omega$.

(ii) For each PEL-structure $Q$ of type $\Omega$, there exists one and only one point $u \in U$ such that $Q$ is isomorphic to $Q_{u}$.

(iii) $U$ and $W$ are Zariski open subset of projective varieties and $f$ is a morphism of varieties.

(iv) There exists a number field $k_{\Omega}$ such that $U, W$ and $f$ are defined over $k_{\Omega}$.

(v) For each $\sigma \in \operatorname{Aut}(\mathbf{C})$ there exists a PEL-type $\Omega^{\sigma}$ and an isomorphism of $\mathscr{F}(\Omega)^{\sigma}$ to $\mathscr{F}\left(\Omega^{\sigma}\right)$ defined over $k_{\Omega^{\sigma}}$.

Proof. See [12, Theorem 5.3] and [14, Proposition 3.1]; see also [5, $\S 8]$.

Theorem 6.2. Let $\Omega$ be as in Theorem 6.1 , and let $f: W \rightarrow U$ be a family of abelian varieties associated to $\Omega$. Let $f^{\prime}: W^{\prime} \rightarrow U^{\prime}$ be another family of abelian varieties such that each fiber $\left(f^{\prime}\right)^{-1}(u)$ for $u \in U$ has a PEL-structure $Q_{u}^{\prime}$. Suppose that at least one $Q_{u}^{\prime}$ is of type $\Omega$ and that the family $\left\{Q_{u}^{\prime} \mid u \in U\right\}$ of PEL-structures satisfies the property (ii) in Theorem 6.1. Then there is a biregular isomorphism between $f: W \rightarrow U$ and $f^{\prime}: W^{\prime} \rightarrow U^{\prime}$.

Proof. See [12, Theorem 5.4 and Theorem 5.5].

Theorem 6.3. If $\pi: Y \rightarrow X$ is a Kuga fiber variety and $\sigma \in \operatorname{Aut}(\mathbf{C})$, then the conjugate $\pi^{\sigma}: Y^{\sigma} \rightarrow X^{\sigma}$ is also a Kuga fiber variety.

Proof. Let $G_{s}=\operatorname{Sp}(V, \beta)(\mathbf{R})$. We shall apply Theorem 5.2 to the equivariant pair

$$
\rho: G \rightarrow G_{S}, \quad \tau: D \rightarrow \mathscr{H}
$$

and the morphism $\phi: X \rightarrow X_{S}$ of arithmetic varieties. For $\sigma \in$ $\operatorname{Aut}(\mathbf{C})$, we consider the conjugate morphism

$$
\phi^{\sigma}: X^{\sigma} \rightarrow X_{S}^{\sigma}
$$


and its lifting $\tau^{\sigma}: D^{\sigma} \rightarrow D_{S}^{\sigma}$. Since $\Gamma_{S}$ and $H$ can be obtained from an admissible and normal PEL-type (see [14, $\S 4-\S 6]), X_{S}^{\sigma}$ is isomorphic to $\Gamma_{S}^{(\sigma)} \backslash \mathscr{H}^{\prime}$ where $\mathscr{H}^{\prime}=\mathscr{H}$ and $\Gamma_{S}^{(\sigma)}$ is an arithmetic subgroup of $\left(G_{S}\right)_{0}^{\sigma}$, the connected component of the identity of $\operatorname{Aut}\left(D_{S}^{\sigma}\right)$ (see e.g. $[14,(3.2)])$. Let

$$
\pi_{S}^{(\sigma)}: Y_{S}^{(\sigma)} \rightarrow X_{S}^{\sigma}
$$

be the standard family of abelian varieties obtained from $\Gamma_{S}^{(\sigma)}, \mathscr{H}$ and $\operatorname{Sp}(V, \beta)$. By Theorem 5.2 there exist a Lie group $G_{1}^{\sigma}$ and a homomorphism $\rho_{1}^{\sigma}: G_{1}^{\sigma} \rightarrow\left(G_{S}\right)_{0}^{\sigma}$ such that $\rho_{1}^{\sigma}$ and $\tau^{\sigma}$ are equivariant and $\rho_{1}^{\sigma}\left(\Gamma^{\sigma}\right)$ is contained in $\Gamma_{S}^{(\sigma)}$. Hence we obtain a Kuga fiber variety $\pi^{(\sigma)}: Y^{(\sigma)} \rightarrow X^{\sigma}$ by pulling back the standard family

$$
\pi_{S}^{(\sigma)}: Y_{S}^{(\sigma)} \rightarrow X_{S}^{\sigma}
$$

via the morphism $\phi^{\sigma}: S^{\sigma} \rightarrow X_{S}^{\sigma}$ :

$$
\begin{array}{ccc}
Y^{(\sigma)} & \stackrel{\phi_{Y}^{(\sigma)}}{\longrightarrow} & Y_{S}^{(\sigma)} \\
\pi^{(\sigma)} \downarrow & & \downarrow \pi_{S}^{(\sigma)} \\
X^{\sigma} \stackrel{\phi^{\sigma}}{\longrightarrow} X_{S}^{\sigma}
\end{array}
$$

On the other hand, by applying $\sigma$ to the commutative diagram

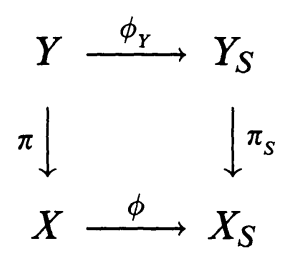

we obtain the following commutative diagram:

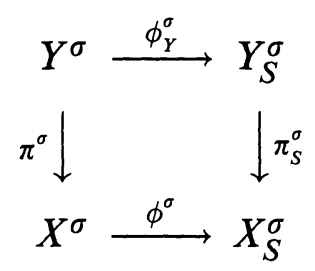

If $\pi_{S}: Y_{S} \rightarrow X_{S}$ is a family of abelian varieties $\mathscr{F}(\Omega)$ associated to a PEL-type $\Omega$, then by Theorem 6.1 the conjugate $\pi_{S}^{\sigma}: Y_{S}^{\sigma} \rightarrow X_{S}^{\sigma}$ is a family $\mathscr{F}\left(\Omega^{\sigma}\right)$ associated to the PEL-type $\Omega^{\sigma}$. Note that the Lie group $\left(G_{S}\right)_{0}^{\sigma}$, the symmetric domain $\mathscr{H}$ and the arithmetic subgroup 
$\Gamma_{S}^{(\sigma)}$ coincide with the corresponding objects associated to the standard family $\pi_{S}^{(\sigma)}: Y_{S}^{(\sigma)} \rightarrow X_{S}^{\sigma}$. If the construction of the fiber variety associated to a PEL-type described in [12, (3.14)] and [13, (5.1)] is used, then $Y_{S}^{\sigma}$ can be constructed in the same way as $Y_{S}^{(\sigma)}$. In particular, at least one fiber of $\pi_{S}^{(\sigma)}: Y_{S}^{(\sigma)} \rightarrow X_{S}^{\sigma}$ has a PEL-structure $\left(Q^{(\sigma)}\right)_{u}$ $\left(u \in X_{S}^{\sigma}\right)$ that is of type $\Omega^{\sigma}$. The standard family $\pi_{S}^{(\sigma)}$ certainly satisfies the property (ii) in Theorem 6.1. To show that the conjugate family $\pi_{S}^{\sigma}: Y_{S}^{\sigma} \rightarrow X_{S}^{\sigma}$ satisfies the same property, let $\left(Q^{\sigma}\right)_{u}$ and $\left(Q^{\sigma}\right)_{v}$ be the PEL-structures of the fibers over $u, v \in X_{S}^{\sigma}$. Then $u$ is equal to $v$ by [13, Proposition 4.4] (see also [15, Theorem 4]); hence the conjugate family $\pi_{S}^{\sigma}$ also satisfies the property (ii) in Theorem 6.1. Thus by Theorem 6.2 there is a biregular isomorphism between the families $\pi_{S}^{\sigma}: Y_{S}^{\sigma} \rightarrow X_{S}^{\sigma}$ and $\pi_{S}^{(\sigma)}: Y_{S}^{(\sigma)} \rightarrow X_{S}^{\sigma}$. Therefore there is an isomorphism between the diagram for $\pi^{(\sigma)}: Y^{(\sigma)} \rightarrow X^{\sigma}$ and the one for $\pi^{\sigma}: Y^{\sigma} \rightarrow X^{\sigma}$ given above; hence it follows that $\pi^{\sigma}: Y^{\sigma} \rightarrow X^{\sigma}$ is a Kuga fiber variety.

\section{REFERENCES}

[1] S. Addington, Equivariant holomorphic maps of symmetric domains, Duke Math. J., 55 (1987), 65-88.

[2] A. Ash, D. Mumford, M. Rapoport and Y. S. Tai, Smooth Compactification of Locally Symmetric Varieties, Math. Sci. Press, Brookline, 1975.

[3] W. Baily and A. Borel, Compactification of arithmetic quotients of bounded symmetric domains, Ann. of Math., 84 (1966), 442-528.

[4] A. Borel, Some metric problems on arithmetic quotients of symmetric spaces and an extension theorem, J. Differential Geom., 6 (1972), 543-560.

[5] M. Borovoi, Langlands' conjecture concerning conjugation of Shimura varieties, Selecta Math. Soviet, 3 (1983/84), 3-39.

[6] D. Kazhdan, On arithmetic varieties, in Lie groups and their representations, Halsted, New York, 1975.

[7] _. On arithmetic varieties II, Israel J. Math., 44 (1983), 139-159.

[8] M. Kuga, Fiber Varieties Over a Symmetric Space Whose Fibers are Abelian Varieties I, II, Lect. Notes, Univ. Chicago, 1963/64.

[9] M. Kuga and S. Ihara, Families of families of abelian varieties, in Algebraic number theory, Japan Soc. for Prom. Sci., Tokyo, 1977.

[10] J. Milne, The action of an automorphism of $\mathbf{C}$ on a Shimura variety and its special points, in Progr. Math., Vol. 35, Birkhäuser, Boston, 1983.

[11] I. Satake, Algebraic Structures of Symmetric Domains, Princeton Univ. Press, 1980.

[12] G. Shimura, Moduli and fiber systems of abelian varieties, Ann. of Math., 83 (1966), 294-338.

[13] _ On the field of definition for a field of automorphic functions II, Ann. of Math., 81 (1965), 124-165. 
[14] G. Shimura, On the field of definition for a field of automorphic functions III, Ann. of Math., 83 (1966), 377-385.

[15] _ Moduli of abelian varieties and number theory, in Proc. Sympos. Pure Math., Vol. 9, Amer. Math. Soc., Providence, RI, 1966.

Received August 31, 1989 and in revised form March 15, 1990.

UNIVERSITY OF NORTHERN IOWA

Cedar Falls, IA 50614 


\section{PACIFIC JOURNAL OF MATHEMATICS EDITORS}

\author{
V. S. VARADARAJAN \\ (Managing Editor) \\ University of California \\ Los Angeles, CA 90024-1555-05 \\ Herbert Clemens \\ University of Utah \\ Salt Lake City, UT 84112 \\ THOMAS ENRIGHT \\ University of California, San Diego \\ La Jolla, CA 92093
}

R. FINN

Stanford University

Stanford, CA 94305

HeRmann FlaschKa

University of Arizona

Tucson, AZ 85721

VAUGHAN F. R. JoNES

University of California

Berkeley, CA 94720

STEVEN KERCKHOFF

Stanford University

Stanford, CA 94305
C. C. MOORE

University of California

Berkeley, CA 94720

MaRTIN SCHARLEMANN

University of California

Santa Barbara, CA 93106

HAROLD STARK

University of California, San Diego

La Jolla, CA 92093

\section{ASSOCIATE EDITORS}
R. ARENS
E. F. BECKENBACH
B. H. NeumanN
F. WolF
K. YosHIDA

\section{SUPPORTING INSTITUTIONS}

UNIVERSITY OF ARIZONA

UNIVERSITY OF BRITISH COLUMBIA

CALIFORNIA INSTITUTE OF TECHNOLOGY

UNIVERSITY OF CALIFORNIA

MONTANA STATE UNIVERSITY

UNIVERSITY OF NEVADA, RENO

NEW MEXICO STATE UNIVERSITY

OREGON STATE UNIVERSITY
UNIVERSITY OF OREGON

UNIVERSITY OF SOUTHERN CALIFORNIA

STANFORD UNIVERSITY

UNIVERSITY OF HAWAII

UNIVERSITY OF TOKYO

UNIVERSITY OF UTAH

WASHINGTON STATE UNIVERSITY

UNIVERSITY OF WASHINGTON 


\section{Pacific Journal of Mathematics}

Vol. 149, No. $1 \quad$ May, 1991

Takao Akahori and Harunori Ameku, On the Romanov kernel and Kuranishi's $L^{2}$-estimate for $\bar{\partial}_{\mathrm{b}}$ over a ball in the strongly pseudo convex

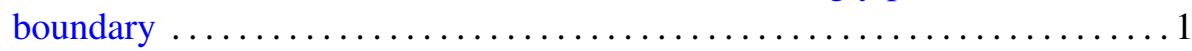

Robert Emile Beaudoin, The proper forcing axiom and stationary set

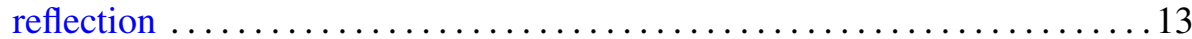

Hans Engler, A matrix Volterra integrodifferential equation occurring in

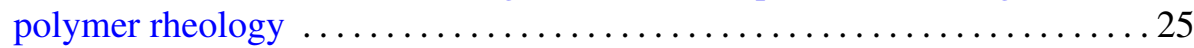

Zhong Ge, On a constrained variational problem and the spaces of

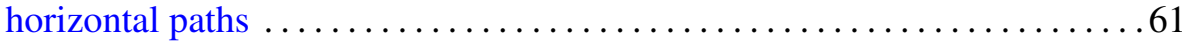

Yutaka Hemmi, Higher homotopy commutativity of $H$-spaces and the

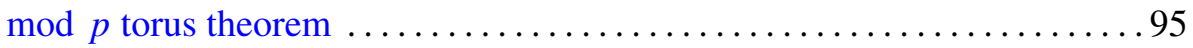

G. D. Johnson, An intrinsic characterization of a class of minimal surfaces

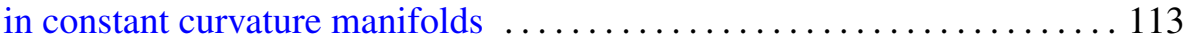

Min Ho Lee, Conjugates of equivariant holomorphic maps of symmetric domains

Jacek Nikiel, H. Murat Tuncali and Edward D. Tymchatyn, On the rim-structure of continuous images of ordered compacta

Tara Lynn Smith, Generalized Clifford-Littlewood-Eckmann groups . . . . . 157

Tara Lynn Smith, Generalized Clifford-Littlewood-Eckmann groups II:

Linear representations and applications 185 